\title{
Subcutaneous Emphysema and Pneumomediastinum Following Oro-Facial First Degree Burns
}

\author{
Ahmad Jaafar ${ }^{\mathrm{a}, \mathrm{d}}$, Abrar Al-Hashemi ${ }^{\mathrm{b}}$, Eman Al-Hashemi ${ }^{\mathrm{c}}$
}

\begin{abstract}
First degree oro-facial burns, particularly by hot liquids, are common in children, and usually heal without complications. Rarely, potentially serious complications that can lead to cardiorespiratory compromise and even death can occur. We present a 15-month-old girl who developed spontaneous subcutaneous emphysema and pneumomediastinum 3 days after sustaining a first degree oro-facial burn by a hot liquid, with a possible thermal inhalational injury. Two important diagnoses that needed to be ruled out are the injury of an air-containing structure, like the pharynx, larynx, trachea, esophagus and bronchial tree as well as necrotizing fasciitis. Literature review revealed a lack of medical research that focuses on the association between such complications and first degree oro-facial burns, particularly in toddlers. The study reveals that spontaneous subcutaneous emphysema and pneumomediastinum can complicate first degree oro-facial burns in toddlers. Our aims are to draw the attention to the possible association between superficial oro-facial burns and the development of spontaneous subcutaneous emphysema and pneumomediastinum, and to encourage further studies to investigate into this association.
\end{abstract}

Keywords: Oro-facial burn; Subcutaneous emphysema; Pneumomediastinum; Toddler

\section{Introduction}

First degree oro-facial burns, especially from hot liquids, are common in children. They usually heal with simple supportive measures without complications. Rarely, potentially serious complications can arise, that might lead to cardiorespiratory compromise and even death, if not detected and managed ear-

\footnotetext{
Manuscript accepted for publication March 20, 2015

a Department of Pediatrics, Mubarak Al-Kabeer Hospital, Kuwait

${ }^{b}$ Department of General Surgery, Al-Amiri Hospital, Kuwait

'Faculty of Medicine, Kuwait University, Kuwait

${ }^{\mathrm{d} C}$ Corresponding Author: Ahmad Jaafar, Department of Pediatrics, Mubarak

Al-Kabeer Hospital, Kuwait. Email: dr.a.jaafar@gmail.com
}

doi: http://dx.doi.org/10.14740/ijcp203w ly. Such complications include subcutaneous emphysema and pneumomediastinum.

Despite the extensive research available in the medical literature about subcutaneous emphysema and pneumomediastinum, limited information is available regarding the occurrence of such complications following first degree oro-facial burns, particularly in toddlers.

\section{Case Report}

A 15-month-old girl, previously healthy, presented to the pediatric emergency department (ED) with first degree burns affecting the nostrils, tip of the nose, chin and oral cavity after accidental immersion of the face in hot soup.

After the accident, the child was taken immediately to the nearest primary health center, in which first aid measures were performed, after which she was sent home. About $2 \mathrm{~h}$ after the accident, she developed a difficulty in breathing and stridor, for which she was brought to the pediatric ED. In the ED examination room, the baby was irritable and crying with evidence of superficial first degree burns affecting the nostrils, tip of the nose, chin and oral cavity. Her vital signs were as follows: temperature was $37.3^{\circ} \mathrm{C}$, respiratory rate was $46 / \mathrm{min}$, heart rate was 110/min, blood pressure was $90 / 64 \mathrm{~mm} \mathrm{Hg}$ and oxygen saturation was $99 \%$ on room air. She was in mild respiratory distress with an audible soft stridor; however, on auscultation, her chest was clear with normal vascular breathing heard all over. The rest of the examination was unremarkable.

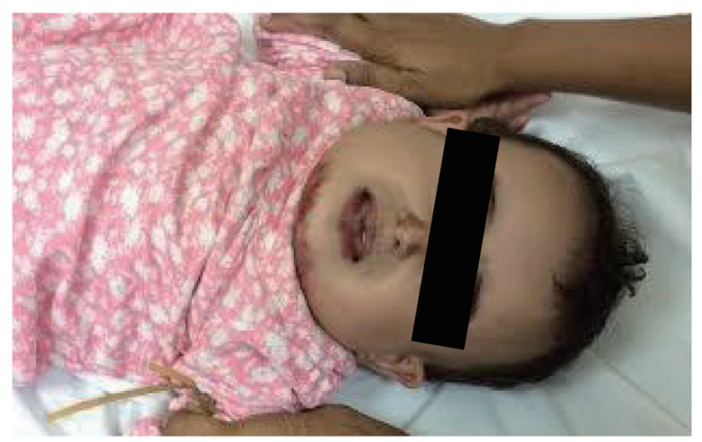

Figure 1. The patient's picture showing facial swelling, taken about $3-4 \mathrm{~h}$ from its onset. 


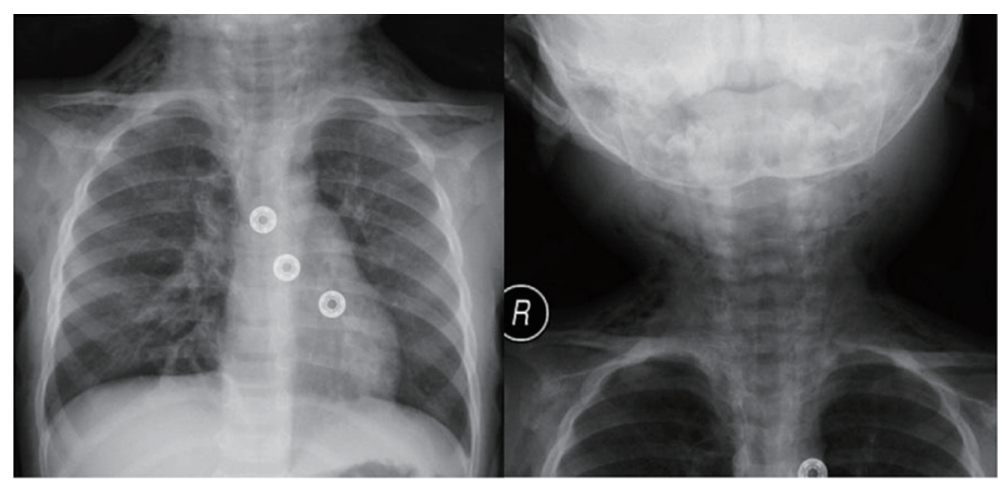

Figure 2. Posterio-anterior chest and neck X-ray films showing cervical subcutaneous emphysema.

She was given a diagnosis of an upper airway inhalational injury, admitted and treated accordingly. She was started on intravenous (IV) hydration, IV antibiotics, IV hydrocortisone, adrenaline nebulization (one nebulization given) and topical preparations for the burns.

The patient improved gradually with the treatment. Three days later, she developed progressive swelling of the face, neck and upper chest (Fig. 1). The patient became irritable; however, she did not develop a stridor or respiratory distress. The vital signs were within normal limits. When palpating the swelling, a crepitus was felt. Routine blood workup including complete blood count (CBC), serum urea and electrolytes (U/E) and coagulation profile was ordered; apart from mild leukocytosis, the results of the blood workup were within normal limits. Neck and chest X-ray was done, showing subcutaneous emphysema in the neck region (Fig. 2, 3). Urgent neck and chest computed tomography (CT) scan with IV contrast was done, which showed extensive subcutaneous emphysema of the neck and chest as well as pneumomediastinum (Fig. 4, 5). The trachea and esophagus appeared intact with no evi-

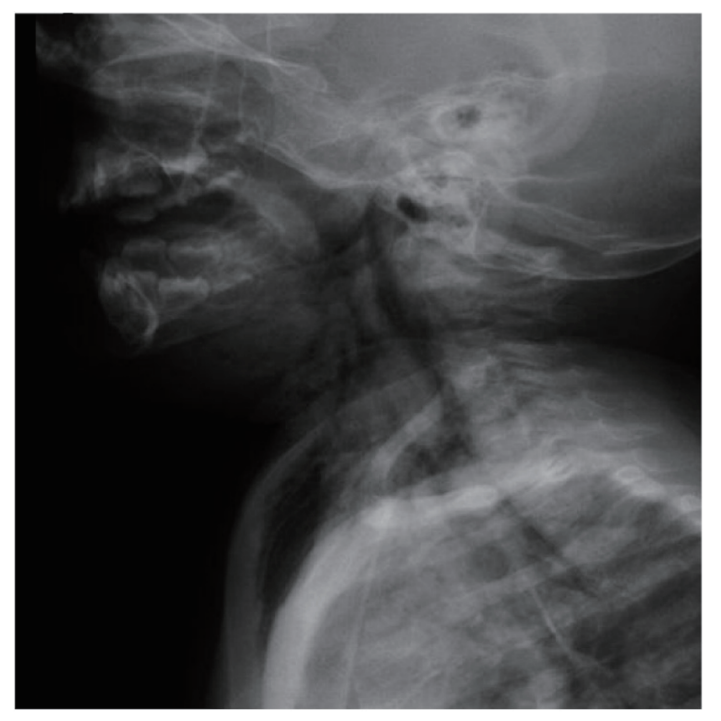

Figure 3. Lateral chest and neck X-ray film showing cervical subcutaneous emphysema as well as subcutaneous emphysema in the upper chest region. dence of perforation. The child was shifted to the pediatric intensive care unit (ICU) in a stable condition.

An otolaryngologist was consulted urgently. Fiber-optic laryngoscopy was done showing no evidence of laryngeal perforation. A pediatric general surgeon was consulted urgently, and after a thorough clinical assessment and review of the imaging studies, necrotizing fasciitis (NF) was suspected. The plan was to perform urgent subcutaneous exploration, obtain a tissue biopsy and perform urgent surgical debridement if indicated.

The patient was shifted to the main operating theater (OT) for subcutaneous exploration under general anesthesia. In the OT, the skin, subcutaneous tissues and fascia were divided, revealing air locules subcutaneously. No discharge or signs of NF were noted. A tissue biopsy was obtained for histopathological examination and culture, after which the operation was terminated, the baby was shifted to the recovery room and then to the pediatric ICU in a stable condition.

Following the operation, the swelling in the patient's face, neck and upper chest improved gradually over 3 - 4 days. After 1 week in the pediatric ICU, the patient was shifted to the general pediatric ward. A repeat neck and chest X-ray showed significant improvement. The histopathology report showed no evidence of infection and the biopsy culture showed no growth after 5 days of incubation. After a few days, the patient was discharged home in a stable condition, with follow-up under general pediatrics.

\section{Discussion}

Subcutaneous emphysema is defined as the presence of air in the subcutaneous tissues, while pneumomediastinum is defined as the presence of air in the mediastinum. It was hypnotized that air can spread from the subcutaneous tissues, particularly in the neck or chest region, to the mediastinum along the fascial planes and vice versa. In fact, pneumomediastinum can be suspected by the presence of subcutaneous emphysema in the neck region [1].

Subcutaneous emphysema is usually an asymptomatic condition. However, certain clinical features can occur with subcutaneous emphysema, which vary according to its location as well as severity. These include disfigurement and discomfort/pain affecting the involved body part(s). Cervical sub- 


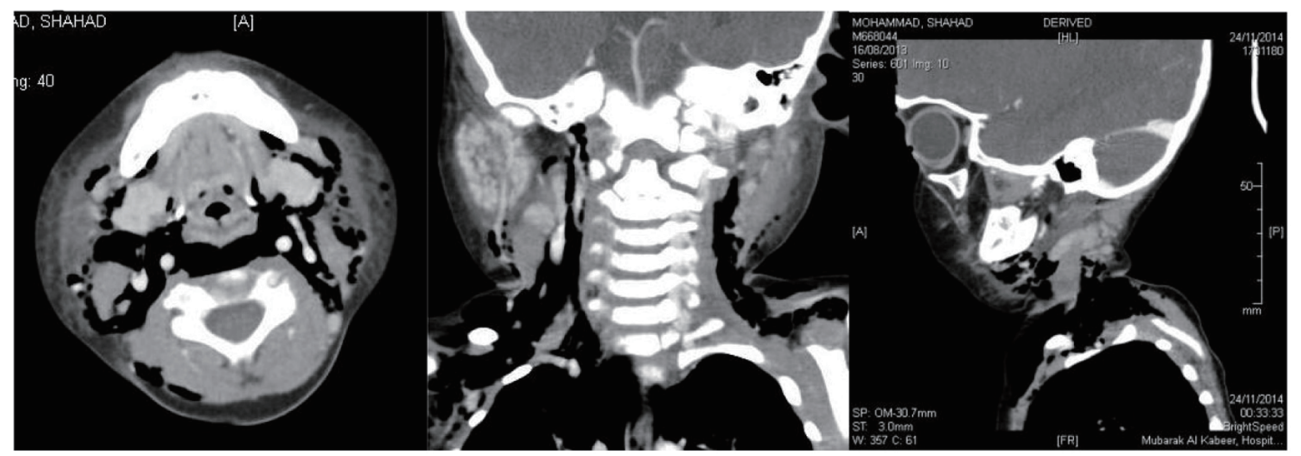

Figure 4. Sagittal, coronal and lateral neck CT scan with IV contrast films showing cervical subcutaneous emphysema.

cutaneous emphysema can result in stridor or life-threatening compression of the trachea [2]. In addition, facial subcutaneous emphysema can track into the orbit and compress the eyeball, threatening vision $[3,4]$. When palpating the subcutaneous emphysema, a crepitus can be felt.

Like subcutaneous emphysema, pneumomediastinum is often asymptomatic. However, clinical features arise when the pressure in the mediastinum increases suddenly or excessively. Complications include 1) pneumothorax, 2) tension pneumomediastinum leading to cardiac temponade and 3) impedance of pulmonary vascular flow by air within the vascular sheaths [5].

The etiologies that have been investigated in this case include the injury of an air-containing structure like the pharynx [6], larynx [7], trachea [8, 9], esophagus and bronchial tree [10]. If any of the previous structures is injured, air can leak from the structure and spread into the subcutaneous tissues and/or mediastinum, giving rise to subcutaneous emphysema and/or pneumomediastinum respectively.

Although the patient sustained a relatively small oro-facial first degree burn, which is defined as a superficial burn limited to the epidermis, a thermal inhalational burn, which is defined as a thermal injury to the airways and lungs from the inhalation of heated gases or hot liquids, most probably occurred. The clues in this patient that point to a thermal inhalational burn include the mechanism though which the burn occurred (face

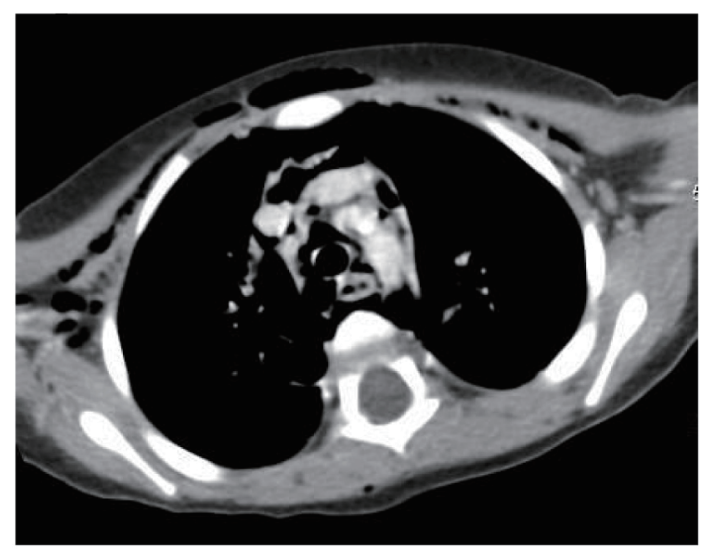

Figure 5. Sagittal chest CT scan with IV contrast film showing pneumomediastinum and subcutaneous emphysema in the anterior chest wall. immersion into a hot liquid) and the occurrence of a stridor shortly after the burn.

Additionally, swallowing a hot liquid can cause a thermal injury to the esophagus. From what is mentioned above, it was necessary to include an injury to an air-containing structure in the deferential diagnosis of this patient.

However, the fact that the subcutaneous emphysema and pneumomediastinum occurred 3 days following the burn makes such etiologies less likely. Additionally, the imaging studies and fiber-optic laryngoscopy excluded the presence of any tear or rupture in any of the above structures.

Another possible etiology that should be considered is NF. $\mathrm{NF}$ is a severe, life-threatening, rapidly progressive soft tissue infection, primarily involving the subcutaneous tissues and underlying fascia [11]. It is associated with systemic toxicity and high mortality [12].

In NF, pain in the affected site is usually the presenting symptom. It is often severe and out of proportion with the physical findings [13]. Moreover, constitution symptoms like fever, usually high-grade fever, anorexia and malaise often accompany the disease. The patients usually appear toxic or severely ill [14]. Local skin features like skin warmth, redness, swelling and tenderness often occur in NF, making it difficult sometimes to distinguish it from cellulitis [13]. Air-formation, giving rise to subcutaneous emphysema, can occur in NF if the organisms causing the infection are gas-forming organisms. This is why a crepitus can be felt when palpating the affected area [15].

It is well established in the medical literature that NF can be caused by trauma even if it is minor, which disturbs the function of the skin and mucus membranes as a barrier against invading organisms. Based on the previous association between trauma and NF, one can associate burns with NF similarly. For instance, Cho et al [13] report a 55-year-old woman, who developed NF, 12 days following a small burn on her right foot. Unfortunately, the medical literature lacks sufficient research regarding this association in children.

In this case, NF was included in the deferential diagnosis because of its close association with burns, the time-frame in which the subcutaneous emphysema and pneumomediastinum occurred (3 days after the burn), elevated WBC count, as well as the seriousness of the diagnosis, making early detection and treatment a priority. However, the absence of fever, particularly high-grade fever and the toxic or ill-looking appearance 
of the child make this diagnosis more distant. In addition, the imaging studies and subcutaneous exploitation that were performed excluded NF.

When no specific etiology is found, the patient is said to have spontaneous subcutaneous emphysema and pneumomediastinum.

\section{Conclusion}

Spontaneous subcutaneous emphysema and pneumomediastinum can complicate oro-facial first degree burns in toddlers. The main deferential diagnosis that needs to be ruled out is the injury of an air-containing structure like the pharynx, larynx, trachea, esophagus and bronchial tree, particularly if the burn is associated with a thermal inhalational injury. Another diagnosis that needs to be considered is NF. Surgical incisions of the subcutaneous tissues and fascia may be curative. Further studies need to be conducted to investigate the casual relationship between superficial oro-facial burns and such complications.

\section{Competing Interests}

The author(s) declare that they have no competing interests.

\section{Abbreviations}

IV: intravenous; CT: computed tomography; CBC: complete blood count; U/E: urea and electrolytes; ED: emergency department; ICU: intensive care unit; OT: operating theater; NF: necrotizing fasciitis

\section{References}

1. Jabourian Z, McKenna EL, Feldman M. Spontaneous pneumomediastinum and subcutaneous emphysema. J Otolaryngol. 1988;17(1):50-53.

2. Abu-Omar Y, Catarino PA. Progressive subcutaneous emphysema and respiratory arrest. J R Soc Med.
2002;95(2):90-91.

3. Singh M, Phua VM, Sundar G. Sight-threatening orbital emphysema treated with needle decompression. Clin Experiment Ophthalmol. 2007;35(4):386-387.

4. Monaghan AM, Millar BG. Orbital emphysema during air travel: a case report. J Craniomaxillofac Surg. 2002;30(6):367-368.

5. Gilhotra R, Malik SK. Pneumomediastinum complicating status asthmaticus. Lung India. 1986;14:35-37.

6. Tixier H, Rattin C, Dunand A, Peaupardin Y, Douvier S, Sagot P, Mourtialon P. Hamman's syndrome associated with pharyngeal rupture occurring during childbirth. Acta Obstet Gynecol Scand. 2010;89(3):407-408.

7. de Bree R, Van Nieuwkerk EB, Vos A, Ekkelkamp S, Sibarani-Ponsen RD, Haasnoot K, Mahieu HF. [Rupture of larynx or trachea resulting from injuries sustained at birth]. Ned Tijdschr Geneeskd. 1999;143(30):1564-1568.

8. Gries CJ, Pierson DJ. Tracheal rupture resulting in lifethreatening subcutaneous emphysema. Respir Care. 2007;52(2):191-195.

9. Carbognani P, Bobbio A, Cattelani L, Internullo E, Caporale D, Rusca M. Management of postintubation membranous tracheal rupture. Ann Thorac Surg. 2004;77(2):406409.

10. Kaloud H, Smolle-Juettner FM, Prause G, List WF. Iatrogenic ruptures of the tracheobronchial tree. Chest. 1997;112(3):774-778.

11. Bingol-Kologlu M, Yildiz RV, Alper B, Yagmurlu A, Ciftci E, Gokcora IH, Ince E, et al. Necrotizing fasciitis in children: diagnostic and therapeutic aspects. J Pediatr Surg. 2007;42(11):1892-1897.

12. Manders SM. Infectious disease update. Dermatol Clin. 2001;19(4):749-756.

13. Yong Suk Cho, Hyeong Tae Yang, et al. Necrotizing fasciitis following a small burn. J Korean Surg Soc. 2010;79:71-74.

14. Stamenkovic I, Lew PD. Early recognition of potentially fatal necrotizing fasciitis. The use of frozen-section biopsy. N Engl J Med. 1984;310(26):1689-1693.

15. Wong $\mathrm{CH}$, Chang HC, Pasupathy S, Khin LW, Tan JL, Low CO. Necrotizing fasciitis: clinical presentation, microbiology, and determinants of mortality. J Bone Joint Surg Am. 2003;85-A(8):1454-1460. 